\title{
基于指数效用无差异价值过程的不完备市场下的 可违约期权的定价模型
}

\author{
王恺明 (1)*, 张徽燕 (2), 姚瑾 ${ }^{(1)}$ \\ (1) 西南财经大学天府学院, 绵阳 621000 ; \\ (2) 电子科技大学经济与管理学院, 成都 610054 \\ E-mail: wkm.6870@163.com, zhanghy@uestc.edu.cn, yaoj@tf-swufe.net
}

收稿日期: 2012-11-14；接受日期: 2013-03-29; * 通信作者

\begin{abstract}
摘要 本文研究不完备市场情况下的可违约期权的动态指数效用无差异定价. 不同于大多数的可违约 期权定价文献, 本文没有假定鞅的不变性, 即通常的 $\mathcal{H}$ 假设, 而是通过信息流的扩张和测度的变换, 将 信用风险敏感的资产转换为一个 $\mathbb{G}$ 局部鞅, 其后引入一个具体的倒向随机微分方程 (BSDE), 并证明 该方程解的存在性与唯一性; 然后利用无差异价值过程 $C_{t}(B, \alpha)$ 在最小摘鞅测度下对一般的投资策 略为上鞅, 而在最优投资策略下为鞅的事实, 证明无差异价值过程 $C_{t}(B, \alpha)$ 就是 $\mathrm{BSDE}$ 的解, 从而给 出可违约期权的定价.
\end{abstract}

关键词 可违约期权 指数效用无差异价值过程 信用敏感资产 倒向随机微分方程

MSC (2010) 主题分类 $60 \mathrm{H} 15,91 \mathrm{~B} 25,91 \mathrm{G} 40$

\section{1 引言}

金融衍生品的定价一直是金融数学领域研究的热点问题. 随着金融创新的发展, 金融衍生品的种 类越来越多，如期权、互换、外汇合约和抵押债券等. 金融衍生品的出现为投资者提供了巨大的投资 机会, 但同时也带来了巨大的金融风险. 在 2007 年发生的次贷危机和最近的欧洲主权债务危机中, 我 们都能看到这些金融衍生品的身影. 在次贷危机中, 衍生品的信用违约扮演了重要作用, 其中的典型就 是抵押债券. 金融衍生品的违约风险就成了人们必须考虑的问题, 而在金融衍生品定价研究中最为广 泛的是期权的定价, 因此, 本文采用指数效用函数最大化的方法对可违约期权的定价作了详细的研究.

在完备市场下对期权的定价，人们一般采用对冲的方法来完成，即构建一个投资组合来对冲风险. 在完备市场环境下, 等价鞅测度是唯一存在的, 因此, 投资组合可以完美复制, 消除风险. 但是在不完 备市场下, 等价鞅测度不唯一, 风险不能完全消除, 只能使风险尽量最小. 人们常常采用二次效用函数 和指数效用函数最优化的方法来完成.

利用二次效用函数, 即均值方差对冲方法给期权定价, 一般有两种, 第一种是投影的方法, 其核心 是寻找最优方差鞅测度. 有很多学者作了深入研究, 如 Schweizer ${ }^{[1,2]}$, Delbaen 和 Schachermayer ${ }^{[3]}$ 对 连续半鞅的情形作了研究, Arai ${ }^{[4]}$, Cerny 和 Kallsen ${ }^{[5]}$ 对具有跳过程的半鞅给出了期权的定价公式, 但是这种方法极其复杂, 尤其是处理带跳的过程. 另外一种是利用倒向随机微分方程的方法来处理衍 生品的定价问题, 这方面的文献异常丰富, 如文献 [6-9] 用均值方差对冲方法对不完备市场下的连续 
过程的期权定价进行了详细的研究, Kohlmann 等人 [10] 和 Jeanbalnc 等人 ${ }^{[11]}$ 对存在跳的半鞅过程的 期权定价也作了研究, 另外, Xiong 和 Kohlmann ${ }^{[12]}$ 还用同样方法对存在跳的债券的定价也作相应的 研究.

除了二次效用函数外, 人们还利用指数效用函数给期权定价. 这种方法首先构建一个投资组合的 倒向随机微分方程和一个与期权有关的倒向随机微分方程, 然后利用二者之差在最优投资策略下为鞅 的事实, 给出与期权有关的倒向随机微分方程的生成元的具体结构, 最后证明方程的解的唯一性和存 在性, 从而解决期权的定价问题. 该定价方法给出的期权价值过程有时也称为指数效用无差异价值过 程, 首先是由 Hodges 和 Neuberger ${ }^{[13]}$ 提出, 随后文献 [14-18] 用这种方法对连续半鞅的期权和债券 定价. Morlalis ${ }^{[19]}$, Xiong 和 Kohlman ${ }^{[20]}$ 还将此方法应用于具有跳的期权和债券的定价.

对于具有信用违约风险的期权定价, 即通常所说的可违约期权, 人们也采用类似方法. 在不完备 市场下, Jeanblanc 等人 ${ }^{[21]}$ 用均值方差对冲和 Hamilton Jacobi Bellman (HJB) 方程对可违约期权的 定价作了研究. Biagini 和 Cretarola ${ }^{[22]}$, Kohlmann 和 Xiong ${ }^{[23]}$ 用均值方差对冲针对不同情形下的可 违约的期权定价作了研究. Kharroubi 等人 ${ }^{[24]}$ 利用鞅最优原则, 构建了三个具体的倒向随机微分方程, 用均值方差对冲方法, 给出可违约期权的定价方程. 在上述可违约期权的定价研究中, 人们通常要求 鞅在空间扩张过程中保持不变的假设, 但这在金融实践中很难维持. 其次在使用指数效用函数定价方 法中涉及的倒向随机微分方程的生成元不满足 Lipschitz 条件, 也不满足二次增长条件, 人们较少采用. 因此, 本文尝试利用指数效用方法对可违约期权进行定价.

本文的具体结构: 第 2 节给出后面定价所需要的预备知识; 第 3 节给出可违约期权的倒向随机微 分方程的解的存在性和唯一性.

\section{2 预备知识}

我们考虑一个有限的时间水平 $T>0$ 和一个完备的概率空间 $\left(\Omega, \mathbb{F}=\left\{\mathcal{F}_{t}\right\}_{0 \leqslant t \leqslant T}, P\right)$, 其中 $\mathbb{F}$ $=\left(\mathcal{F}_{t}\right)_{0 \leqslant t \leqslant T}$ 满足通常的条件, 即是右连续的和完备的, 且 $\mathcal{F}_{0}$ 包含了 $\mathbb{F}$ 所有的零测集, 同时假设 $\mathbb{F}$ 为 连续流.

\section{1 信用违约风险建模}

我们现在考虑信用违约的情形. 我们认为信用违约事件发生的时间 $\tau$ 是个非负的随机变量, 满足 $P(\tau=0)=0$, 且 $P(\tau>t)>0$ 对任意时间 $t \in \mathbb{R}_{+}$. 我们引入一个右连续的过程 $D$, 其中 $D_{t}=1_{\tau \leqslant t}$, 用 $\mathbb{D}$ 表示由 $D_{t}$ 生成的 $\sigma$ 域: $\mathcal{D}_{t}=\sigma\left(D_{u}: u \leqslant t\right)$. 令 $\mathbb{G}=\mathbb{D} \vee \mathbb{F}$, 也就是说, $\mathcal{G}_{t}=\mathcal{D}_{t} \vee \mathcal{F}_{t}, \mathbb{G}=\left(\mathcal{G}_{t}\right)_{t \geqslant 0}$ 对任意时间 $t$ 都成立. 这样通过流的扩张, 我们就有一个更大的概率空间 $(\Omega, \mathcal{G}, P)$, 后面的研究均在 此空间进行. 对于信用违约事件, 我们首先给出如下假设.

假设 2.1 信用违约事件发生时间 $\tau$ 是一个首达时间, 即存在一个随机过程族 $\left(\rho^{u}, u \in \mathbb{R}_{+}\right)$, 使 得对任意 $u>0$, 随机过程 $\left(\rho_{t}^{u}, 0 \leqslant t \leqslant T\right)$ 是一个 $\mathbb{F}$ 鞅, 且对于任意 $\xi \geqslant 0$ 和 $t \leqslant T$, 有

$$
P\left(\tau>\xi \mid \mathcal{F}_{t}\right)=\int_{\xi}^{\infty} \rho_{t}^{u} d u
$$

假设 2.1 可参见文献 $[25$, 假设 H1]. 另外我们还定义 Azema 上鞅

$$
G_{t}:=P\left(\tau>t \mid \mathcal{F}_{t}\right)
$$


对该上鞅我们有其 Doob-Meyer 分解

$$
G_{t}=\int_{t}^{\infty} \rho_{t}^{u} d u=\int_{0}^{\infty} \rho_{t \wedge u}^{u} d u-\int_{0}^{t} \rho_{u}^{u} d u=J_{t}-A_{t},
$$

其中 $J_{t}$ 是鞅, $A_{t}$ 是其可料补偿, 同时也是过程 $D_{t}$ 的可料补偿. 令 $L_{t}$ 为 $D_{t}$ 的 Doob-Meyer 分解的鞅 部分, 则 $L_{t}$ 可写成

$$
L_{t}=D_{t}-\int_{0}^{t} \frac{\rho_{u}^{u}}{G_{u-}} 1_{\{u \leqslant \tau\}} d u,
$$

对于 $\mathbb{F}$ 停时 $\varepsilon$ 与 $\mathbb{G}$ 停时 $\tau$ 之间的关系, 我们需要作如下假设.

假设 2.2 信用事件发生停时 $\tau$ 非 $\mathbb{F}$ 停时 $\varepsilon$, 即 $P[\tau=\varepsilon]=0$.

假设 2.2 是信用风险建模的基本假设, 同时在此假设下可料补偿 $A_{t}$ 是一个连续过程 ${ }^{[25-27]}$. 从而, 鞅 $L_{t}$ 为

$$
L_{t}=D_{t}-\int_{0}^{t} \frac{\rho_{u}^{u}}{G_{u}} 1_{\{u \leqslant \tau\}} d u .
$$

为了书写方便, 我们令 $\lambda_{u}:=\frac{\rho_{u}^{u}}{G_{u-}}$, 从而, $L_{t}$ 可以写成

$$
L_{t}=D_{t}-\int_{0}^{t} \lambda_{u} 1_{u \leqslant \tau} d u
$$

接下来考虑一个 $\mathbb{R}^{d}$ 值 $\mathbb{F}$ 资产 $S$, 它为无信用风险资产, 即

$$
d S_{t}=b_{t} d t+\varsigma_{t} d S_{t}^{*}+N_{t},
$$

其中 $S^{*}$ 和 $N$ 为 $\mathbb{R}^{d}$ 值 $(\mathbb{F}, P)$ 连续鞅, 且 $S^{*}$ 与 $N$ 强正交, 即 $\left\langle S^{*}, N\right\rangle=0, b$ 为 $\mathbb{R}^{d}$ 值可料过程. 由文 献 $[27$, 定理 3.1] 可知,

$$
\widehat{S}=S^{*}-\eta \cdot\left\langle S^{*}\right\rangle, \quad \widehat{N}=N-V,
$$

$\widehat{S}$ 和 $\widehat{N}$ 为 $(\mathbb{G}, P)$ 局部鞅, $\eta \cdot\left\langle S^{*}\right\rangle$ 中的. 表示为 $\int \eta d S^{*}$ (下同), 其中 $\eta$ 和 $V$ 为

$$
\eta_{s}=\frac{1-D_{s}}{G_{s}} z_{s}+\frac{D_{s}}{G_{s}} a_{s}^{\tau}, \quad V_{t}=\frac{1-D}{G} \cdot\langle N, G\rangle_{t}+\frac{D}{\rho^{u}}\left\langle N, \rho^{u}\right\rangle_{t},
$$

具体可参见文献 [27]. 在不完备市场下, 对于信用敏感资产 $S$ 可以改写为

$$
S_{t}=\int_{0}^{t} \mu_{u} d u+\varsigma \cdot \widehat{S}_{t}+\zeta \cdot L_{t}+\widehat{N}_{t}
$$

其中 $\varsigma_{t}$ 和 $\zeta_{t}$ 为 $\mathbb{R}^{d \times d}$ 值 $\mathbb{G}$ 可料过程. 对于该资产我们作如下假设.

假设 2.3 $\varsigma_{t}$ 和 $\zeta_{t}$ 为 $\mathbb{R}^{d \times d}$ 值 $\mathbb{G}$ 可料过程, 且满足 $\mu_{t}$ 和 $\varsigma_{t}$ 有界, $\varsigma_{t}$ 可逆, $-1<\zeta_{t} \leqslant C$.

我们定义信用敏感资产 $S$ 的等价鞅测度 $Q$, 其密度过程为

$$
Z_{T}=\left.\frac{d Q}{d P}\right|_{\mathcal{G}_{T}}=\mathcal{E}_{T}\left(\varphi \cdot \widehat{S}+\psi \cdot L+N^{\perp}\right),
$$

其中 $\varphi$ 和 $\psi$ 为 $\mathbb{R}^{d}$ 值 $\mathbb{G}$ 可料有界过程, 且 $\psi>-1 . N^{\perp}$ 为 $(\mathbb{G}, P)$ 均方可积鞅, 与 $(\widehat{S}, L)$ (强) 正交. 为了使 $S$ 在测度 $Q$ 下为局部鞅, 我们有引理 2.4. 
引理 2.4 在测度概率 $Q$ 下, $Z S$ 为 $S$ 局部鞅当且仅当

$$
\int_{0}^{t} \mu_{u} d u+\int_{0}^{t} \varphi_{u}^{\mathrm{T}} \varsigma_{u} d u+\int_{0}^{t} \psi_{u}^{\mathrm{T}} \zeta_{u} \lambda_{u} 1_{u \leqslant \tau} d u+\left\langle N^{\perp}, \widehat{N}\right\rangle_{t}=0 .
$$

证明 由 Itô 公式可知, $Z S$ 为 $S$ 局部鞅当且仅当 $S+\frac{1}{Z} \cdot[S, Z]$ 为局部鞅,

$$
\begin{aligned}
S_{t}+\frac{1}{Z_{t}} \cdot[S, Z]_{t}= & \int_{0}^{t} \mu_{u} d u+\varsigma \cdot \widehat{S}_{t}+\zeta \cdot L_{t}+\widehat{N}_{t}+\int_{0}^{t} \varphi_{u}^{\mathrm{T}} \varsigma_{u} d u \\
& +\psi_{t}^{\mathrm{T}} \zeta_{t} L_{t}+\int_{0}^{t} \psi_{u}^{\mathrm{T}} \zeta_{u} \lambda_{u} 1_{u \leqslant \tau} d u+\left\langle N^{\perp}, \widehat{N}\right\rangle_{t} \\
= & \int_{0}^{t} \mu_{u} d u+\int_{0}^{t} \varphi_{u}^{\mathrm{T}} \varsigma_{u} d u+\int_{0}^{t} \psi_{u}^{\mathrm{T}} \zeta_{u} \lambda_{u} 1_{u \leqslant \tau} d u+\left\langle N^{\perp}, \widehat{N}\right\rangle_{t} \\
& +\varsigma \cdot \widehat{S}_{t}+\zeta \cdot L_{t}+\widehat{N}_{t}+\psi_{t}^{\mathrm{T}} \zeta_{t} \cdot L_{t},
\end{aligned}
$$

其中 $\mathrm{T}$ 表示向量和矩阵的转置, 从而, 有

$$
\int_{0}^{t} \mu_{u} d u+\int_{0}^{t} \varphi_{u}^{\mathrm{T}} \varsigma_{u} d u+\int_{0}^{t} \psi_{u}^{\mathrm{T}} \zeta_{u} \lambda_{u} 1_{u \leqslant \tau} d u+\left\langle N^{\perp}, \widehat{N}\right\rangle_{t}=0 .
$$

由此可知, 在概率测度 $Q$ 下, 由 Girsanov 定理可知,

$$
\widetilde{S}_{t}=\widehat{S}_{t}-\int_{0}^{t} \varphi_{1, u} d u, \quad \widetilde{L}_{t}=L_{t}-\int_{0}^{t} \psi_{u}^{\mathrm{T}} \lambda_{u} 1_{u \leqslant \tau} d u, \quad \widetilde{N}=\widehat{N}-\left\langle\widehat{N}, N^{\perp}\right\rangle
$$

均为 $(\mathbb{G}, Q)$ 鞅, 从而, $S$ 可改写成

$$
S_{t}=\varsigma \cdot \widetilde{S}_{t}+\zeta \cdot \widetilde{L}_{t}+\widetilde{N}_{t}
$$

引理 2.4 得证.

\section{2 指数效用函数}

在指数效用的可违约期权的定价中, 由于我们考虑的市场是不完备的, 因此, 等价鞅测度是不唯 一的, 我们需要求出定价所需要的最小熵鞅测度. 在此首先给出最小熵鞅测度的定义.

定义 2.5 令概率测度 $Q$ 和测度 $P$ 绝对连续, 其密度过程为

$$
Z=\left.\frac{d Q}{d P}\right|_{\mathcal{G}_{T}},
$$

则相对熵 $H(Q \mid P)$ 为

$$
H(Q \mid P)=E\left(\frac{d Q}{d P} \ln \frac{d Q}{d P}\right), \quad Q \ll P,
$$

否则 $H(Q \mid P)=+\infty$. 最小熵鞅测度 $Q^{*}$ 就是下述最优化问题的解,

$$
\inf _{Q \in \mathbb{P}_{e, l}} H(Q \mid P)=H\left(Q^{*} \mid P\right) .
$$

相对熵, 有时也称为 Kullback-Leiber 过程或者 Entropy-Hellinger 过程, 其中

$$
\mathbb{P}_{e}:=\{Q \sim P \mid S \text { 为 } Q \text { 局部鞅 }\}, \mathbb{P}_{e, l}:=\left\{Q \in \mathbb{P}_{e} \mid H(Q \mid P)<\infty\right\} .
$$


接下来考虑指数效用函数, 它具有如下形式:

$$
U(x)=-\exp (-\alpha x)
$$

其中 $\alpha \in(0, \infty)$ 为风险厌恶系数. 对于可违约期权 $B$, 为 $\mathbb{R}$ 值有界 $\mathcal{G}_{T \wedge \tau}$ 可测随机变量, 具有如下 形式:

$$
B \in L^{\infty}\left(\mathcal{G}_{T \wedge \tau}, P\right), \quad B=B^{a} 1_{\tau>T}+B^{b} 1_{\tau \leqslant T},
$$

其中 $B^{a}$ 为 $\mathbb{R}$ 值 $\mathcal{F}_{T}$ 可测随机变量, $B^{b}$ 为 $\mathbb{R}$ 值 $\mathbb{F}$ 适应过程, 且满足

$$
\left\|B^{a}\right\|_{\infty}<+\infty, \quad\left\|\sup _{t \in[0, T]}\left|B_{t}^{b}\right|\right\|_{\infty}<+\infty
$$

$\|\cdot\|_{\infty}$ 表示本性下确界, $|\cdot|$ 表示 Euclid 范数 (下同). 对可违约期权 $B$ 定价, 我们采用指数效用无 差异方法来解决这个问题. 在给定指数效用函数和初始投资资金 $x_{t}$ 时, 我们用一个有界的右连左极 (RCLL) 的半鞅 $C_{t}(B, \alpha)$ 来表示未定权益的价值, 它满足

$$
\underset{\vartheta \in \Theta}{\operatorname{ess} \sup } E\left[U\left(x+X_{t, T \wedge \tau}(\vartheta)\right) \mid \mathcal{G}_{t}\right]=\sup _{\vartheta \in \Theta} E\left[U\left(x+C_{t}(B, \alpha)+X_{t, T \wedge \tau}(\vartheta)-B\right) \mid \mathcal{G}_{t}\right],
$$

其中 $X_{t, T \wedge \tau}(\vartheta)$ 为投资组合, $\vartheta$ 为投资策略, $\Theta$ 为可取投资策略, 具体表达式为

$$
X_{t, T \wedge \tau}(\vartheta)=x_{t}+\int_{t}^{T \wedge \tau} \vartheta_{u} d S_{u}, \quad \Theta:=\left\{\vartheta \mid \vartheta \mathbb{R}^{d} \mathbb{G} X(\vartheta)\right\}
$$

为了后面的定价处理, 我们假定可取投资策略 $\Theta$ 为紧集. 对指数效用无差异价值过程, 我们有如下 命题.

命题 2.6 无差异指数效用价值过程 $C_{t}(B, \alpha)$, 可以表示为

$$
C_{t}(B, \alpha)=\frac{1}{\alpha} \ln \underset{\vartheta}{\operatorname{essinf}} E_{Q^{*}}\left[\mathrm{e}^{\alpha\left(B-X_{t, T}(\vartheta)\right)} \mid \mathcal{G}_{t}\right], \quad 0 \leqslant t \leqslant T .
$$

证明 命题 2.6 的证明过程参见文献 [17, 命题 3].

\section{3 可违约未定权益的定价}

我们引入 BSDE, 该方程在可违约期权的定价中起着核心作用. 该方程具体表达式为

$$
\begin{aligned}
& Y_{t}=Y_{0}+\int_{0}^{t} f\left(u, \theta_{1}, \theta_{2}, \widetilde{N}^{\perp}\right) d u+\int_{0}^{t} \theta_{1, u} d \widetilde{S}_{u}+\int_{[0, t]} \theta_{2, u} d \widetilde{L}_{u}+\widetilde{N}_{t}^{\perp}, \\
& Y_{T \wedge \tau}=B
\end{aligned}
$$

其中 $\theta_{1}$ 和 $\theta_{2}$ 为 $\mathbb{R}^{d}$ 值有界 $\mathbb{G}$ 可料过程, $\widetilde{N}^{\perp}$ 为 $\mathbb{R}$ 值局部鞅, 与 $\widetilde{S}$ 和 $\widetilde{L}$ 强正交, 即 $\left\langle\widetilde{S}, \widetilde{N}^{\perp}\right\rangle=0$, $\left\langle\widetilde{L}, \widetilde{N}^{\perp}\right\rangle=0$. 方程的解为 $\left(Y, \theta_{1}, \theta_{2}, \widetilde{N}^{\perp}\right)$, 其中生成元 $f\left(t, \theta_{1}, \theta_{2}, \widetilde{N}^{\perp}\right)$ 具有如下形式:

$$
\begin{aligned}
f\left(t, \theta_{1}, \theta_{2}, \widetilde{N}^{\perp}\right) d t:= & \underset{\vartheta \in \Theta}{\operatorname{esinf}}\left\{\frac{\alpha}{2}\left(\theta_{1, t}-\vartheta_{t}^{\mathrm{T}} \varsigma_{t}\right)^{\mathrm{T}} d\langle\widetilde{S}\rangle_{t}\left(\theta_{1, t}-\vartheta_{t}^{\mathrm{T}} \varsigma_{t}\right)+\frac{\alpha}{2} d\left\langle\left(\vartheta \cdot \widetilde{N}-\widetilde{N}^{\perp}\right)\right\rangle_{t}\right. \\
& \left.+\left(\frac{\mathrm{e}^{\alpha\left(\theta_{2, t}-\vartheta_{t}^{\mathrm{T}} \zeta_{t}\right)}-1-\alpha\left(\theta_{2, t}-\vartheta_{t}^{\mathrm{T}} \zeta_{t}\right)}{\alpha}\right)^{\mathrm{T}} \lambda_{t} 1_{t \leqslant \tau} d t\right\} .
\end{aligned}
$$


为研究倒向随机微分方程的解, 我们需要定义相应的空间:

(1) $\mathcal{S}_{\mathbb{G}}^{\infty}$ 表示 $\mathbb{R}$ 值 $\mathbb{G}$ 适应的右连左极本性有界过程 $\left(Y_{t}\right)_{t \in[0, T]}$ :

$$
\|Y\|_{\mathcal{S}^{\infty}}=\left\|\sup _{t \in[0, T]}\left|Y_{t}\right|\right\|_{\infty} \leqslant+\infty ;
$$

(2) $L_{\mathbb{G}}^{2}$ 表示 $\mathbb{R}^{d}$ 值 $\mathbb{G}$ 可测可料过程 $\left(\theta_{1, t}\right)_{t \in[0, T]}$, 满足

$$
\left\|\theta_{1}\right\|_{L^{2}}=\left(E\left(\int_{0}^{t} \theta_{1, u}^{\mathrm{T}} d\langle\widetilde{S}\rangle_{u} \theta_{1, u}\right)\right)^{\frac{1}{2}}<+\infty
$$

(3) $L_{\perp}^{2}$ 表示 $\mathbb{R}$ 值 $\mathbb{G}$ 可测过程 $\left(\left(\widetilde{N}^{\perp}\right)_{t}\right)_{t \in[0, T]}$, 满足

$$
\left\|\widetilde{N}^{\perp}\right\|_{L^{2}}=\left(E\left(\int_{0}^{t} d\left\langle\widetilde{N}^{\perp}\right\rangle_{u}\right)\right)^{\frac{1}{2}}<+\infty ;
$$

(4) $L^{2}(\lambda)$ 表示 $\mathbb{R}^{d}$ 值 $\mathbb{G}$ 可测可料过程 $\left(\theta_{2, t}\right)_{t \in[0, T]}$, 满足

$$
\left\|\theta_{2}\right\|_{L^{2}}=\left(E\left(\int_{0}^{T \wedge \tau} \theta_{2, u}^{\mathrm{T}} \lambda_{u} \theta_{2, u} d u\right)\right)^{\frac{1}{2}}<+\infty .
$$

方程的解的唯一性与 BMO (bounded mean oscillation) 鞅有密切的联系, 因此, 我们首先给出 BMO 鞅的定义, 该定义具体可参见何声武等人 ${ }^{[28]}$ 的半鞅与随机分析.

定义 $3.1 \mathrm{BMO}$ 鞅空间定义为 $\mathcal{H}^{2}$ 空间的一个子空间, 对一个 $K \in \mathcal{H}^{2}$ 的局部鞅, 如果存在一个 常数 $k$, 使得对任意 $\mathbb{F}$ 停时 $\varepsilon$ 有 $E\left[\left(K_{T}-K_{\varepsilon-}\right)^{2} \mid \mathcal{F}_{\varepsilon}\right] \leqslant k$, 则局部鞅 $K$ 称为 BMO 鞅, $\|K\|_{\text {BMO }}$ 代表 最小的 $k$, 如果它存在的话.

对连续鞅 $K$, 有 $E\left[\left(\langle K\rangle_{T}-\langle K\rangle_{\varepsilon}\right)^{2} \mid \mathcal{F}_{\varepsilon}\right] \leqslant k$, 对于具有跳的鞅还需 $|\Delta K|_{\varepsilon}<\infty$, 对于 (3.1) 的生成 元 $f\left(t, \theta_{1}, \theta_{2}, \widetilde{N}^{\perp}\right)$, 我们有如下估计, 即引理 3.2 .

引理 3.2 假设 (3.1) 的生成元具有 (3.2) 的形式, 则存在常数 $C_{1}$ 和 $C_{2}$ 使得生成元满足

$$
\begin{aligned}
& 0 \leqslant f\left(t, \theta_{1}, \theta_{2}, \widetilde{N}^{\perp}\right) \leqslant \frac{\alpha}{2} \theta_{1}^{\mathrm{T}} \theta_{1}+\frac{\alpha}{2}\left\langle\widetilde{N}^{\perp}\right\rangle_{t}+\left(\frac{\mathrm{e}^{\alpha \theta_{2}}-1-\alpha \theta_{2}}{\alpha}\right)^{\mathrm{T}} \lambda_{t} 1_{t \leqslant \tau}, \\
& \left|f\left(t, \theta_{1}^{1}, \theta_{2}, \widetilde{N}^{\perp}\right)-f\left(t, \theta_{1}^{2}, \theta_{2}, \widetilde{N}^{\perp}\right)\right| \leqslant C_{1}\left(1+\left|\theta_{1}^{1}\right|+\left|\theta_{1}^{2}\right|\right)\left|\theta_{1}^{1}-\theta_{1}^{2}\right|, \\
& \left|f\left(t, \theta_{1}, \theta_{2},\left(\widetilde{N}^{\perp}\right)^{1}\right)-f\left(t, \theta_{1}, \theta_{2},\left(\widetilde{N}^{\perp}\right)^{2}\right)\right| \leqslant C_{2}\left(1+\left|\left(\widetilde{N}^{\perp}\right)^{1}\right|+\left|\left(\widetilde{N}^{\perp}\right)^{2}\right|\right)\left|\left(\widetilde{N}^{\perp}\right)^{1}-\left(\widetilde{N}^{\perp}\right)^{2}\right|, \\
& f\left(t, \theta_{1}, \theta_{2}^{1}, \widetilde{N}^{\perp}\right)-f\left(t, \theta_{1}, \theta_{2}^{2}, \widetilde{N}^{\perp}\right) \leqslant \chi\left(s, \theta_{2}^{1}, \theta_{2}^{2}\right)\left(\theta_{2}^{1}-\theta_{2}^{2}\right) \lambda_{t} 1_{t \leqslant \tau} .
\end{aligned}
$$

证明 由生成元的定义, 显然, 有

$$
0 \leqslant f\left(t, \theta_{1}, \theta_{2}, \widetilde{N}^{\perp}\right) \leqslant \frac{\alpha}{2} \theta_{1, t}^{\mathrm{T}}\langle\widetilde{S}\rangle_{t} \theta_{1, t}+\frac{\alpha}{2}\left\langle\widetilde{N}^{\perp}\right\rangle_{t}+\left(\frac{\mathrm{e}^{\alpha \theta_{2}}-1-\alpha \theta_{2}}{\alpha}\right)^{\mathrm{T}} \lambda_{t} 1_{t \leqslant \tau} .
$$

对过程 $\theta_{1}$ 而言, 存在一个常数 $C_{1}$, 对任意 $\theta_{1}^{1}, \theta_{1}^{2} \in \mathrm{BMO}[\widetilde{S}]$, 则有

$$
\left|f\left(t, \theta_{1}^{1}, \theta_{2}, \widetilde{N}^{\perp}\right)-f\left(t, \theta_{1}^{2}, \theta_{2}, \widetilde{N}^{\perp}\right)\right| \leqslant C_{1}\left(1+\left|\theta_{1}^{1}\right|+\left|\theta_{1}^{2}\right|\right)\left|\theta_{1}^{1}-\theta_{1}^{2}\right| .
$$

对鞅 $\widetilde{N}^{\perp}$ 而言, 存在一个常数 $C_{2}$, 对任意 $\theta_{1} \in \mathrm{BMO}[\widetilde{S}], \theta_{2}$ 有界, $\left(\widetilde{N}^{\perp}\right)^{1}$ 和 $\left(\widetilde{N}^{\perp}\right)^{2}$ 均与 $\mathrm{BMO}$ 鞅 $\widetilde{S}$ 强 正交, 则有

$$
\left|f\left(t, \theta_{1}, \theta_{2},\left(\widetilde{N}^{\perp}\right)^{1}\right)-f\left(t, \theta_{1}, \theta_{2},\left(\widetilde{N}^{\perp}\right)^{2}\right)\right| \leqslant C_{2}\left(1+\left|\left(\widetilde{N}^{\perp}\right)^{1}\right|+\left|\left(\widetilde{N}^{\perp}\right)^{2}\right|\right)\left|\left(\widetilde{N}^{\perp}\right)^{1}-\left(\widetilde{N}^{\perp}\right)^{2}\right| .
$$


对过程 $\theta_{2}$ 而言, 对任意 $\theta_{1} \in \mathrm{BMO}[\widetilde{S}], \widetilde{N}^{\perp}$ 与 $\mathrm{BMO}$ 鞅 $\widetilde{S}$ 强正交, 且 $\theta_{2}^{1}$ 和 $\theta_{2}^{2}$ 非负有界, 则有

$$
\begin{aligned}
f & \left(t, \theta_{1}, \theta_{2}^{1}, \widetilde{N}^{\perp}\right)-f\left(t, \theta_{1}, \theta_{2}^{2}, \widetilde{N}^{\perp}\right) \\
& \leqslant \underset{\vartheta \in \Theta}{\operatorname{essinf}}\left\{\left(\frac{\mathrm{e}^{\alpha\left(\theta_{2}^{1}-\vartheta^{\prime} \zeta\right)}-1-\alpha\left(\theta_{2}^{1}-\vartheta^{\mathrm{T}} \zeta\right)}{\alpha}\right)^{\mathrm{T}} \lambda_{t} 1_{t \leqslant \tau}-\left(\frac{\mathrm{e}^{\alpha\left(\theta_{2}^{2}-\vartheta^{\mathrm{T}} \zeta\right)}-1-\alpha\left(\theta_{2}^{2}-\vartheta^{\mathrm{T}} \zeta\right)}{\alpha}\right)^{\mathrm{T}} \lambda_{t} 1_{t \leqslant \tau}\right\} \\
& =\underset{\vartheta \in \Theta}{\operatorname{essinf}}\left(\frac{\mathrm{e}^{\alpha\left(\theta_{2}^{1}-\vartheta^{\mathrm{T}} \zeta\right)}-\mathrm{e}^{\alpha\left(\theta_{2}^{2}-\vartheta^{\mathrm{T}} \zeta\right)}-\alpha\left[\left(\theta_{2}^{1}-\vartheta^{\mathrm{T}} \zeta\right)-\left(\theta_{2}^{2}-\vartheta^{\mathrm{T}} \zeta\right)\right]}{\alpha}\right)^{\mathrm{T}} \lambda_{t} 1_{t \leqslant \tau} .
\end{aligned}
$$

令 $g_{\alpha}(y)=\frac{\mathrm{e}^{\alpha y}-1-\alpha y}{\alpha}$, 则有

$$
\begin{aligned}
& f\left(t, \theta_{1}, \theta_{2}^{1}, \widetilde{N}^{\perp}\right)-f\left(t, \theta_{1}, \theta_{2}^{2}, \widetilde{N}^{\perp}\right) \\
& \quad \leqslant \underset{\vartheta \in \Theta}{\operatorname{esinf}}\left\{\int_{0}^{1} g_{\alpha}^{\prime \prime}\left(\gamma\left(\theta_{2}^{1}-\vartheta^{\mathrm{T}} \zeta\right)+(1-\gamma)\left(\theta_{2}^{2}-\vartheta^{\prime} \zeta\right)\right) d \gamma\right\}\left(\theta_{2}^{1}-\theta_{2}^{2}\right) \lambda_{t} 1_{t \leqslant \tau},
\end{aligned}
$$

其中 $\gamma \in[0,1], g_{\alpha}^{\prime \prime}(\cdot)$ 表示对 $\gamma$ 求导数, $\theta_{2}^{1}-\theta_{2}^{2}=\left(\theta_{2}^{1}-\theta_{2}^{2}\right) 1_{\theta_{2}^{1} \geqslant \theta_{2}^{2}}-\left(-\left(\theta_{2}^{1}-\theta_{2}^{2}\right)\right) 1_{\theta_{2}^{1}<\theta_{2}^{2}}$. 再令

$$
\begin{aligned}
\chi\left(t, \theta_{2}^{1}, \theta_{2}^{2}\right):= & \underset{\vartheta \in \Theta}{\operatorname{esssup}}\left\{\int_{0}^{1} g_{\alpha}^{\prime \prime}\left(\gamma\left(\theta_{2}^{1}-\vartheta^{\mathrm{T}} \zeta\right)+(1-\gamma)\left(\theta_{2}^{2}-\vartheta^{\mathrm{T}} \zeta\right)\right) d \gamma\right\} 1_{\theta_{2}^{1}<\theta_{2}^{2}} \\
& -\underset{\vartheta \in \Theta}{\operatorname{essinf}}\left\{\int_{0}^{1} g_{\alpha}^{\prime \prime}\left(\gamma\left(\theta_{2}^{1}-\vartheta^{\mathrm{T}} \zeta\right)+(1-\gamma)\left(\theta_{2}^{2}-\vartheta^{\mathrm{T}} \zeta\right)\right) d \gamma\right\} 1_{\theta_{2}^{1} \geqslant \theta_{2}^{2}} .
\end{aligned}
$$

从而有

$$
f\left(t, \theta_{1}, \theta_{2}^{1}, \widetilde{N}^{\perp}\right)-f\left(t, \theta_{1}, \theta_{2}^{2}, \widetilde{N}^{\perp}\right) \leqslant\left\{\chi\left(t, \theta_{2}^{1}, \theta_{2}^{2}\right)\left(\theta_{2}^{1}-\theta_{2}^{2}\right)\right\}^{\mathrm{T}} \lambda_{t} 1_{t \leqslant \tau} .
$$

由假设可知, $\theta_{2}^{1}$ 和 $\theta_{2}^{2}$ 均为非负有界过程, 存在一个常数 $C_{3}$, 使得

$$
-1<\chi\left(t, \theta_{2}^{1}, \theta_{2}^{2}\right) \leqslant C_{3} .
$$

从而引理 3.2 得证.

对于 (3.1) 的解 $\left(Y, \theta_{1}, \theta_{2}, \widetilde{N}^{\perp}\right)$ 的有界性, 我们有估计 (3.11) 和 (3.12), 而且方程的解的鞅部分 $\int_{0}^{t} \theta_{1, u} d \widetilde{S}_{u}, \int_{[0, t]} \theta_{2, u} d \widetilde{L}_{u}$ 和 $\widetilde{N}_{t}^{\perp}$ 均为 BMO 鞅, 我们有命题 3.3 .

命题 3.3 对于生成元 $f$ 满足上述条件, 对 (3.1) 的解 $\left(Y, \theta_{1}, \theta_{2}, \widetilde{N}^{\perp}\right)$, 有

$$
\begin{aligned}
& C_{4} \leqslant|Y|_{t} \leqslant C_{5}, \\
& E_{Q^{*}}\left(\left(\int_{\varepsilon}^{T \wedge \tau} \theta_{1, s}^{2} d s+\int_{\varepsilon}^{T \wedge \tau} \theta_{2, s}^{2} d s\right) \mid \mathcal{G}_{\varepsilon}\right) \leqslant C_{6}, \\
& \int_{0}^{t} \theta_{1, u} d \widetilde{S}_{u}, \int_{[0, t]} \theta_{2, u} d \widetilde{L}_{u}, \widetilde{N}_{t}^{\perp} \text { 均为 BMO 鞅. }
\end{aligned}
$$

证明 对任意 $\mathbb{G}$ 停时 $\varepsilon$, 我们对 $\mathrm{e}^{\kappa Y_{t}}, t \in[\varepsilon, T \wedge \tau]$ 应用 Itô 公式, 有

$$
d \mathrm{e}^{\alpha Y_{t}}=\alpha \mathrm{e}^{\alpha Y_{t}} d Y_{t}+\frac{\alpha^{2}}{2} \mathrm{e}^{\alpha Y_{t}} d\langle Y\rangle_{t}+\left\{\mathrm{e}^{\alpha \Delta Y_{t}}-\alpha \Delta Y_{t}-1\right\} d D_{t},
$$

两端积分, 有

$$
\mathrm{e}^{\alpha Y_{T \wedge \tau}}-\mathrm{e}^{\alpha Y_{\varepsilon}}=-\int_{\varepsilon}^{T \wedge \tau} \alpha \mathrm{e}^{\alpha Y_{t}}\left\{f\left(t, \theta_{1}, \theta_{2}, \widetilde{N}^{\perp}\right)-\frac{\alpha}{2}\left|\theta_{1, t}\right|^{2}-d\langle\widetilde{N}\rangle_{t}-\frac{\left(\mathrm{e}^{\alpha \theta_{2, t}}-\alpha \theta_{2, t}-1\right)}{\alpha} \lambda_{t} 1_{t \leqslant \tau}\right\} d t
$$




$$
\begin{aligned}
& +\int_{\varepsilon}^{T \wedge \tau} \alpha \mathrm{e}^{\alpha Y_{t}} \theta_{1, t} d \widetilde{S}_{t}+\int_{[\varepsilon, T \wedge \tau]} \alpha \mathrm{e}^{\alpha Y_{t}} \theta_{2, t} d \widetilde{L}_{t} \\
& +\int_{\varepsilon}^{T \wedge \tau} \alpha \mathrm{e}^{\alpha Y_{t}} d \widetilde{N}_{t}^{\perp}+\int_{[\varepsilon, T \wedge \tau]} \alpha \mathrm{e}^{\alpha Y_{t}}\left(\mathrm{e}^{\alpha \theta_{2, t}}-\alpha \theta_{2, t}-1\right) d \widetilde{L}_{t} .
\end{aligned}
$$

由于 $\int_{\varepsilon}^{T \wedge \tau} \alpha \mathrm{e}^{\alpha Y_{t}} \theta_{1, t} d \widetilde{S}_{t}$ 等为局部鞅, 取局部化序列 $\varepsilon_{n}$, 使得 $n \rightarrow \infty$, 有 $\varepsilon_{n} \rightarrow T \wedge \tau$, 可知,

$$
\int_{\varepsilon}^{\varepsilon_{n}} \alpha \mathrm{e}^{\alpha Y_{t}} \theta_{1, t} d \widetilde{S}_{t}, \quad \int_{\left[\varepsilon, \varepsilon_{n}\right]} \alpha \mathrm{e}^{\alpha Y_{t}} \theta_{2, t} d \widetilde{L}_{t}
$$

为鞅, 对上式两端取条件期望, 则有

$$
\begin{aligned}
\mathrm{e}^{\alpha Y_{\varepsilon}}-E_{Q^{*}}\left[\mathrm{e}^{\alpha Y_{T \wedge \tau}} \mid \mathcal{G}_{\varepsilon}\right]= & \mathrm{e}^{\alpha Y_{\varepsilon}}-E_{Q^{*}}\left[\mathrm{e}^{\alpha B} \mid \mathcal{G}_{\varepsilon}\right] \\
= & E_{Q^{*}}\left[\int _ { \varepsilon } ^ { T \wedge \tau } \alpha \mathrm { e } ^ { \alpha Y _ { t } } \left\{f\left(t, \theta_{1}, \theta_{2}, \widetilde{N}^{\perp}\right)-\frac{\alpha}{2} \theta_{1, t}^{\mathrm{T}} d\langle\widetilde{S}\rangle_{t} \theta_{1, t}-\frac{\alpha}{2} d\langle\widetilde{N}\rangle_{t}\right.\right. \\
& \left.\left.-\frac{\left(\mathrm{e}^{\alpha \theta_{2, t}}-\alpha \theta_{2, t}-1\right)^{\mathrm{T}}}{\alpha} \lambda_{t} 1_{t \leqslant \tau}\right\} d t \mid \mathcal{G}_{\varepsilon}\right] .
\end{aligned}
$$

由 (3.7) 可知,

$$
f\left(t, \theta_{1}, \theta_{2}, \widetilde{N}^{\perp}\right) d t-\frac{\alpha}{2} \theta_{1, t}^{\mathrm{T}} d\langle\widetilde{S}\rangle_{t} \theta_{1, t}-\frac{\alpha}{2} d\langle\widetilde{N}\rangle_{t}-\frac{\left(\mathrm{e}^{\alpha \theta_{2, t}}-\alpha \theta_{2, t}-1\right)^{\mathrm{T}}}{\alpha} \lambda_{t} 1_{t \leqslant \tau} d t \leqslant 0,
$$

且有

$$
E_{Q^{*}}\left[\mathrm{e}^{\alpha B} \mid \mathcal{G}_{\varepsilon}\right] \leqslant \mathrm{e}^{\alpha\|B\|_{\infty}} .
$$

因此, 取 $C_{2}=\|B\|_{\infty}$, 其中 $\|\cdot\|_{\infty}$ 表示本性上确界, 可得 $Y_{t} \leqslant C_{2}$. 为证 $C_{1} \leqslant Y_{t}$, 由 (3.1), 有

$$
Y_{t}-Y_{T \wedge \tau}=\int_{t}^{T \wedge \tau} f\left(t, \theta_{1}, \theta_{2}, N^{\perp}\right) d t-\int_{t}^{T \wedge \tau} \theta_{1, t} d \widetilde{S}_{t}-\int_{[t, T \wedge \tau]} \theta_{2, t} d \widetilde{L}_{t}-\int_{t}^{T \wedge \tau} d \widetilde{N}_{t}^{\perp}
$$

由于 $\int_{\varepsilon}^{T \wedge \tau} \theta_{1, t} d \widetilde{S}_{t}, \int_{[t, T \wedge \tau]} \theta_{2, t} d \widetilde{L}_{t}$ 为局部鞅, 取局部化序列 $\varepsilon_{n}$ 使得 $n \rightarrow \infty$ 有 $\varepsilon_{n} \rightarrow T \wedge \tau$, 可知,

$$
\int_{\varepsilon}^{\varepsilon_{n}} \theta_{1, t} d \widetilde{S}_{t}, \quad \int_{\left[t, \varepsilon_{n}\right]} \theta_{2, t} d \widetilde{L}_{t}
$$

为鞅. 两端取条件期望, 有

$$
Y_{t}=E_{Q^{*}}\left[Y_{T \wedge \tau} \mid \mathcal{G}_{t}\right]
$$

取 $C_{2}=-\|B\|_{\infty}$, 可得结论.

现在来证明 (3.13), 令 $\left(Y, \theta_{1}, \theta_{2}, \widetilde{N}^{\perp}\right)$ 是方程的解, 对 $Y_{t}^{2}$ 用 Itô 公式, 并积分, 有

$$
\begin{aligned}
Y_{T \wedge \tau}^{2}-Y_{t}^{2}= & 2 \int_{t}^{T \wedge \tau} f\left(u, \theta_{1}, \theta_{2}, \widetilde{N}^{\perp}\right) d u+2 \int_{t}^{T \wedge \tau} Y_{u} \theta_{1, u} d \widetilde{S}_{u}+2 \int_{[t, T \wedge \tau]} Y_{u} \theta_{2, u} d \widetilde{L}_{u} \\
& +\int_{t}^{T \wedge \tau} Y_{u} d \widetilde{N}_{u}^{\perp}+\int_{t}^{T \wedge \tau} \theta_{1, u}^{\mathrm{T}} d\langle\widetilde{S}\rangle_{u} \theta_{1, u}+\int_{t}^{T \wedge \tau} d\left\langle\widetilde{N}^{\perp}\right\rangle_{u} \\
& +\int_{[t, T \wedge \tau]} \theta_{2, u}^{\mathrm{T}} d \widetilde{L}_{u} \theta_{2, u}+\int_{t}^{T \wedge \tau} \theta_{2, u}^{\mathrm{T}} \lambda_{u} \theta_{2, u} 1_{u \leqslant \tau} d u
\end{aligned}
$$


而鞅 $\widetilde{L}$ 的可料二次变差过程和二次变差过程均存在, 且为

$$
[\widetilde{L}]_{t}=D_{t}, \quad d\langle\widetilde{L}\rangle_{t}=\lambda_{t} 1_{t \leqslant \tau} d t .
$$

从而, 上式可写为

$$
\begin{aligned}
Y_{T \wedge \tau}^{2}-Y_{t}^{2}= & 2 \int_{t}^{T \wedge \tau} Y_{u} f\left(u, \theta_{1}, \theta_{2}, \widetilde{N}^{\perp}\right) d u+2 \int_{t}^{T \wedge \tau} Y_{u} \theta_{1, u} d \widetilde{S}_{u} \\
& +2 \int_{[t, T \wedge \tau]} Y_{u} \theta_{2, u} d \widetilde{L}_{u}+\int_{t}^{T \wedge \tau} Y_{u} d \widetilde{N}_{u}^{\perp}+\int_{t}^{T \wedge \tau} \theta_{1, u}^{\mathrm{T}} d\langle\widetilde{S}\rangle_{u} \theta_{1, u} \\
& +\int_{t}^{T \wedge \tau} d\left\langle\widetilde{N}^{\perp}\right\rangle_{u}+\int_{[t, T \wedge \tau]} \theta_{2, u}^{\mathrm{T}} d \widetilde{L}_{u} \theta_{2, u}+\int_{t}^{T \wedge \tau} \theta_{2, u}^{\mathrm{T}} d\langle\widetilde{L}\rangle \theta_{2, u} .
\end{aligned}
$$

由于 $\int_{t}^{T \wedge \tau} Y_{u} \theta_{1, u} d \widetilde{S}_{u}$ 和 $\int_{[t, T \wedge \tau]} Y_{u} \theta_{2, u} d \widetilde{L}_{u}$ 为局部鞅, 取局部化序列 $\varepsilon_{n}$ 使得 $n \rightarrow \infty$, 有 $\varepsilon_{n} \rightarrow T \wedge \tau$, 可 知, $\int_{t}^{\varepsilon_{n}} Y_{u} \theta_{1, u} d \widetilde{S}_{u}$ 和 $\int_{\left[t, \varepsilon_{n}\right]} Y_{u} \theta_{2, u} d \widetilde{L}_{u}$ 为鞅. 对上式两端取条件期望, 在 $Q^{*}$ 和 $\mathcal{G}_{t}$ 下, 由于 $C_{1} \leqslant Y_{t} \leqslant C_{2}$ 和 $f\left(u, \theta_{1}, \theta_{2}, \tilde{N}^{\perp}\right)$ 有界, 从而, 存在一个常数 $C_{3}$, 使得

$$
E_{Q^{*}}\left\{\int_{t}^{T \wedge \tau} \theta_{1, u}^{\mathrm{T}} d\langle\widetilde{S}\rangle_{u} \theta_{1, u} \mid \mathcal{G}_{t}\right\}+E_{Q^{*}}\left\{\int_{t}^{T \wedge \tau} d\left\langle\widetilde{N}^{\perp}\right\rangle_{u} \mid \mathcal{G}_{t}\right\}+E_{Q^{*}}\left\{\int_{t}^{T \wedge \tau} \theta_{2, u}^{\mathrm{T}} d\langle\widetilde{L}\rangle \theta_{2, u} \mid \mathcal{G}_{t}\right\} \leqslant C_{3}^{2},
$$

即

$$
\left\|\theta_{1} \cdot \widetilde{S}\right\|_{\mathrm{BMO}}+\left\|\widetilde{N}^{\perp}\right\|_{\mathrm{BMO}}+\left\|\theta_{2} \cdot \widetilde{L}\right\|_{\mathrm{BMO}} \leqslant C_{3}^{2} .
$$

于是有方程解的鞅部分为 $\mathrm{BMO}$ 鞅, 从而命题得证.

现在来证明 (3.1) 的解的唯一性, 我们有定理 3.4.

定理 3.4 生成元为 $f\left(u, \theta_{1}, \theta_{2}, \widetilde{N}^{\perp}\right)$, 终值为 $B$ 的 (3.1) 具有唯一解

$$
\left(Y, \theta_{1}, \theta_{2}, \widetilde{N}^{\perp}\right) \in \mathcal{S}_{\mathbb{G}}^{\infty} \times L_{\mathbb{G}}^{2} \times L_{\perp}^{2} \times L^{2}(\lambda) .
$$

证明 假定 $(3.1)$ 有两个解 $\left(Y^{1}, \theta_{1}^{1}, \theta_{2}^{1},\left(\widetilde{N}^{\perp}\right)^{1}\right)$ 和 $\left(Y^{2}, \theta_{1}^{2}, \theta_{2}^{2},\left(\widetilde{N}^{\perp}\right)^{2}\right)$, 我们引入

$$
\bar{Y}=Y^{1}-Y^{2}, \quad \bar{\theta}_{1}=\theta_{1}^{1}-\theta_{1}^{2}, \quad \bar{\theta}_{2}=\theta_{1}^{2}-\theta_{2}^{2}, \quad \overline{\widetilde{N}^{\perp}}=\left(\tilde{N}^{\perp}\right)^{1}-\left(\tilde{N}^{\perp}\right)^{2} .
$$

我们对 $\bar{Y}$ 在 $T \wedge \tau$ 与 $t$ 之间积分, 从而, 有

$$
\begin{aligned}
\bar{Y}_{T \wedge \tau}-\bar{Y}_{t}= & \int_{t}^{T \wedge \tau}\left(f\left(u, \theta_{1}^{1}, \theta_{2}^{1}\right)-f\left(u, \theta_{1}^{2}, \theta_{2}^{2}\right)\right) d u \\
& +\int_{t}^{T \wedge \tau} \bar{\theta}_{1, u} d \widetilde{S}_{u}+\int_{[t, T \wedge \tau]} \bar{\theta}_{2, u} d \widetilde{L}_{u}+\int_{t}^{T \wedge \tau} d \widetilde{N}_{u}^{\perp} \\
= & \int_{t}^{T \wedge \tau}\left(f\left(u, \theta_{1}^{1}, \theta_{2}^{1}\right)-f\left(u, \theta_{1}^{2}, \theta_{2}^{1}\right)+f\left(u, \theta_{1}^{2}, \theta_{2}^{1}\right)-f\left(u, \theta_{1}^{2}, \theta_{2}^{2}\right)\right) d u \\
& +\int_{t}^{T \wedge \tau} \bar{\theta}_{1, u} d \widetilde{S}_{u}+\int_{[t, T \wedge \tau]} \bar{\theta}_{2, u} d \widetilde{L}_{u}+\int_{t}^{T \wedge \tau} d \widetilde{N}_{u}^{\perp} \\
= & \int_{t}^{T \wedge \tau} \beta(u) \bar{\theta}_{1} d u+\int_{t}^{T \wedge \tau} \chi\left(u, \theta_{1}^{1}, \theta_{2}^{1}\right) \bar{\theta}_{2} \lambda_{u} 1_{u \leqslant \tau} d u \\
& +\int_{t}^{T \wedge \tau} \bar{\theta}_{1, u} d \widetilde{S}_{u}+\int_{[t, T \wedge \tau]} \bar{\theta}_{2, u} d \widetilde{L}_{u}+\int_{t}^{T \wedge \tau} \overline{d \widetilde{N}_{u}^{\perp}}
\end{aligned}
$$




$$
+\int_{t}^{T \wedge \tau} d \widetilde{\widetilde{N}_{u}^{\perp}}-\int_{t}^{T \wedge \tau} d\left\langle\left(\left(N^{\perp}\right)^{1}-\left(N^{\perp}\right)^{2}\right), \frac{\alpha}{2}\left(\left(N^{\perp}\right)^{1}+\left(N^{\perp}\right)^{2}\right)\right\rangle_{u}
$$

其中

$$
\beta(u):=\frac{f\left(u, \theta_{1}^{1}, \theta_{2}^{1}, \widetilde{N}^{\perp}\right)-f\left(u, \theta_{1}^{2}, \theta_{2}^{1}, \widetilde{N}^{\perp}\right)}{\theta_{1}^{1}-\theta_{1}^{2}},
$$

在此假定 $\frac{0}{0}=0$, 然后我们定义一个测度变换:

$$
d \bar{Q}=\mathcal{E}\left(\beta \cdot \widetilde{S}+\chi \cdot \widetilde{L}+\frac{\alpha}{2}\left(\left(N^{\perp}\right)^{1}+\left(N^{\perp}\right)^{2}\right)_{u}\right)_{T} d Q^{*} .
$$

由定义 3.1 和引理 3.2 的证明可知, $\beta \cdot \widetilde{S}_{T \wedge \tau}, \chi \cdot \widetilde{L}_{T \wedge \tau}$ 和 $\frac{\alpha}{2}\left(\left(N^{\perp}\right)^{1}+\left(N^{\perp}\right)^{2}\right)_{T \wedge \tau}$ ( $\frac{\alpha}{2}$ 为常数) 均为 BMO 鞅, 可知 $\bar{Q}$ 为概率测度. 令

$$
\widetilde{S}^{\beta}=\widetilde{S}-\langle\widetilde{S}, \beta \cdot \widetilde{S}\rangle, \quad \widetilde{L}^{\chi}=\widetilde{L}-\langle\widetilde{L}, \chi \cdot \widetilde{L}\rangle, \quad \overline{\widetilde{N}_{u}^{\perp}}=\overline{\widetilde{N}_{u}^{\perp}}-\left\langle\overline{\widetilde{N}_{u}^{\perp}}, \frac{\alpha}{2}\left(\left(N^{\perp}\right)^{1}+\left(N^{\perp}\right)^{2}\right)\right\rangle .
$$

由 Girsanov 定理和文献 $\left[28\right.$, 定理 10.9] 可知, $\bar{\theta}_{1} \cdot \widetilde{S}^{\beta}$ 和 $\bar{\theta}_{2} \cdot \widetilde{L}^{\chi}$ 为 BMO 鞅,

$$
\bar{Y}_{T \wedge \tau}-\bar{Y}_{t}=\int_{t}^{T \wedge \tau} \bar{\theta}_{1, u} d \widetilde{S}_{u}^{\beta}+\int_{[t, T \wedge \tau]} \bar{\theta}_{2, u} d \widetilde{L}_{u}^{\chi}+\int_{t}^{T \wedge \tau} d \widetilde{N}_{u}^{\perp} .
$$

对上式两端取条件期望, 有

$$
E_{\bar{Q}}\left[\bar{Y}_{T \wedge \tau} \mid \mathcal{G}_{t}\right]=E_{\bar{Q}}\left[\bar{Y}_{t} \mid \mathcal{G}_{t}\right]=\bar{Y}_{t} .
$$

由于 $Y_{T \wedge \tau}^{1}=B=Y_{T \wedge \tau}^{2}$, 从而, 有

$$
\bar{Y}_{t}=0
$$

由 $t$ 的任意性, 定理得证.

为了完成可违约期权的定价, 需要证明 (3.1) 的解的存在性, 我们还需引入鞅最优化原则 (martingale optimality principle), 即引理 3.5 和 3.6.

引理 3.5 假定 $S$ 有界, $\mathbb{P}_{e, l} \neq \emptyset$, 且 $B \in L^{\infty}\left(\mathcal{G}_{T \wedge \tau}, P\right)$, 固定 $\alpha>0$, 则有

(1) 存在一个 RCLL 过程 $J^{B}=\left(J_{t}^{B}\right)_{0 \leqslant t \leqslant T}$ 使得对任意 $t \in[0, T]$, 有

$$
J_{t}^{B}=\underset{\vartheta \in \Theta}{\operatorname{essinf}} E_{Q^{*}}\left[\mathrm{e}^{\alpha\left(B-X_{t, T \wedge \tau}(\vartheta)\right.} \mid \mathcal{G}_{t}\right], \quad Q^{*} \text {-a.s. }
$$

$J_{t}^{B}$ 是 $J$ 的最大 RCLL 过程且 $J_{T \wedge \tau}=\mathrm{e}^{\alpha B}, J \mathrm{e}^{-\alpha X(\vartheta)}$ 对任意 $\vartheta \in \Theta$ 是 $Q^{*}$ 下鞅.

(2) 下列三个性质等价:

(a) $\vartheta^{*} \in \Theta$ 为 $(3.13)$ 的最优解, 即 $J_{0}^{B}=E_{Q^{*}}\left[\mathrm{e}^{\alpha\left(B-X_{T \wedge \tau}\left(\vartheta^{*}\right)\right)}\right]$;

(b) $\vartheta^{*} \in \Theta$ 在任意 $t$ 下都是最优的, 即 $J_{t}^{B}=E_{Q^{*}}\left[\mathrm{e}^{\alpha\left(B-X_{t, T \wedge \tau}\left(\vartheta^{*}\right)\right)} \mid \mathcal{G}_{t}\right]$;

(c) $\vartheta^{*} \in \Theta$ 时, $J \mathrm{e}^{-\alpha X\left(\vartheta^{*}\right)}$ 是 $Q^{*}$ 鞅.

(3) 我们可以选择 $\frac{1}{\alpha} \ln J^{B}$ 作为 $C(B ; \alpha)$ 的 RCLL 版本, 对任意 $\mathbb{G}$ 停时 $\sigma \leqslant \varepsilon \leqslant T$, 有

$$
C_{\sigma}(B, \alpha)=\frac{1}{\alpha} \underset{\vartheta \in \Theta}{\ln \operatorname{sinf}} E_{Q^{*}}\left[\mathrm{e}^{\alpha\left(C_{\varepsilon}(B ; \alpha)-X_{t, T \wedge \tau}(\vartheta)\right)} \mid \mathcal{G}_{\sigma}\right] .
$$

证明 参见文献 [17, 定理 12]. 
引理 3.6 假定 $\theta_{1}, \theta_{2}$ 和 $\tilde{N}^{\perp}$ 为 $\mathbb{G}$ 可料过程, $\Theta$ 为紧集, 则有

$$
\begin{aligned}
f\left(t, \theta_{1}, \theta_{2}, \widetilde{N}^{\perp}\right) d t= & \operatorname{essinf}_{\vartheta \in \Theta}\left\{\frac{\alpha}{2}\left(\theta_{1, t}-\vartheta_{t}^{\mathrm{T}} \varsigma_{t}\right)^{\mathrm{T}} d\langle\widetilde{S}\rangle_{t}\left(\theta_{1, t}-\vartheta_{t}^{\mathrm{T}} \varsigma_{t}\right)+\frac{\alpha}{2} d\left\langle\left(\vartheta \cdot \widetilde{N}-\widetilde{N}^{\perp}\right)\right\rangle_{t}\right. \\
& \left.+\left(\frac{\mathrm{e}^{\alpha\left(\theta_{2, t}-\vartheta_{t}^{\mathrm{T}} \zeta_{t}\right)}-1-\alpha\left(\theta_{2, t}-\vartheta_{t}^{\mathrm{T}} \zeta_{t}\right)}{\alpha}\right)^{\mathrm{T}} \lambda_{t} 1_{t \leqslant \tau} d t\right\}
\end{aligned}
$$

仍为 $\mathbb{G}$ 可料过程, 且最优投资策略 $\vartheta^{*}$ 存在, 即 $\vartheta^{*} \in \Theta$.

证明 该引理的思路我们参考了文献 [18, 引理 11] 和 [19, 引理 6]. 对 $d \in \mathbb{N}$, 考虑 $\mathbb{R}^{d}$ 的紧子集, $\mathcal{B}\left(H^{d}\right)$ 为 $H^{d}$ 的 Borel $\sigma$ 域. 则函数 $f\left(t, \theta_{1}, \theta_{2}, \widetilde{N}^{\perp}\right):=f\left(\vartheta_{t}, \theta_{1, t}, \theta_{2, t},\left(\widetilde{N}^{\perp}\right)_{t}\right)$ 为

$$
f\left(\vartheta_{t}, \theta_{1, t}, \theta_{2, t},\left(\tilde{N}^{\perp}\right)_{t}\right): H^{d} \times \mathbb{R}^{d} \times \mathbb{R}^{d} \times \mathbb{R} \rightarrow \mathbb{R}
$$

的映射, 由于 $H^{d}$ 和 $\mathbb{R}^{d}$ 均为标准空间 (具体参见文献 [29, 第二部分]) $\vartheta_{t}, \theta_{1, t}, \theta_{2, t}$ 和 $\left(\widetilde{N}^{\perp}\right)_{t}$ 均 $\mathbb{G}$ 为可料 过程, 都为左连续过程, 因此, 由可测选择定理知, 函数 $f\left(t, \theta_{1}, \theta_{2}, \widetilde{N}^{\perp}\right)$ 为 $\left(\mathcal{B}\left(H^{d}\right) \otimes \mathcal{B}\left(\mathbb{R}^{d}\right) \otimes \mathcal{B}\left(\mathbb{R}^{d}\right) \otimes \mathcal{B}(\mathbb{R})\right.$ $\rightarrow \mathcal{B}(\mathbb{R}))$ 可测. 由于 $\Theta$ 为紧集, 可知, 函数 $f\left(t, \theta_{1}, \theta_{2}, \widetilde{N}^{\perp}\right)$ 可料.

对于最优投资策略存在性的证明与文献 $[19$, 引理 6 的 b] 类似. 构建一个集合

$$
\begin{aligned}
O:= & \left\{(t, \omega)|| f\left(t, \omega, \vartheta_{t}(\omega), \theta_{1, t}(\omega), \theta_{2, t}(\omega),\left(\widetilde{N}^{\perp}\right)_{t}(\omega)\right)\right. \\
& \left.-\underset{\vartheta \in \Theta}{\operatorname{sinf} \inf } f\left(t, \omega, \vartheta_{t}(\omega), \theta_{1, t}(\omega), \theta_{2, t}(\omega),\left(\widetilde{N}^{\perp}\right)_{t}(\omega)\right) \mid+\left(1-1_{\Theta}(\vartheta)\right) \neq 0\right\},
\end{aligned}
$$

其中 $f\left(t, \theta_{1}, \theta_{2}, \widetilde{N}^{\perp}\right):=f\left(t, \omega, \vartheta_{t}(\omega), \theta_{1, t}(\omega), \theta_{2, t}(\omega),\left(\widetilde{N}^{\perp}\right)_{t}(\omega)\right)$, 由于 $f\left(t, \theta_{1}, \theta_{2}, \widetilde{N}^{\perp}\right)$ 为 $\mathbb{G}$ 可料, 从而得

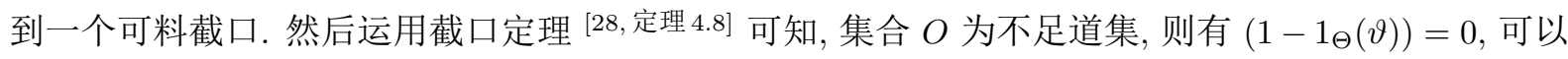
确保 $\vartheta:=\vartheta(t, \omega)$ 在可取投资组合 $\Theta$ 中取值, 从而引理 3.6 得证.

现在来证明方程 (3.1) 的解的存在性, 即无差异指数效用价值过程 $C(B, \alpha)$ 是方程 (3.1) 的解, 这 是我们最主要的结果, 我们有定理 3.7 .

定理 3.7 假定 $\mathbb{P}_{e, l} \neq \emptyset, B \in L^{\infty}\left(\mathcal{G}_{T \wedge \tau}\right)$, 无差异指数效用价值过程 $C(B, \alpha)$ 在最小熵鞅测度 $Q^{*}$ 下, 是方程 (3.1) 的唯一解.

证明 由引理 3.5, 我们知道投资策略 $\vartheta^{*}$ 在最小熵鞅测度 $Q^{*}$ 下 $J \mathrm{e}^{-\alpha X\left(\vartheta^{*}\right)}$ 是 $Q^{*}$ 鞅, 可违约期 权的指数效用无差异价值过程 $C(B, \alpha)$ 可以表示为

$$
C_{t}(B, \alpha)=\frac{1}{\alpha} \ln J_{t}^{B}=\frac{1}{\alpha} \ln \left(J_{t}^{B} \mathrm{e}^{-\alpha X_{t, T \wedge \tau}\left(\vartheta^{*}\right)}\right)+X_{t, T \wedge \tau}\left(\vartheta^{*}\right) .
$$

由 (3.13) 可知,

$$
C_{T \wedge \tau}(B, \alpha)=\frac{1}{\alpha} \ln J_{T \wedge \tau}^{B}=B
$$

而

$$
\begin{aligned}
J_{t}^{B} \mathrm{e}^{-\alpha X_{t, T \wedge \tau}\left(\vartheta^{*}\right)} & =E_{Q^{*}}\left(J_{T \wedge \tau}^{B} \mathrm{e}^{-\alpha X_{T \wedge \tau, T \wedge \tau}\left(\vartheta^{*}\right)} \mid \mathcal{G}_{t}\right)=E_{Q^{*}}\left(J_{T \wedge \tau}^{B} \mid \mathcal{G}_{t}\right) \\
& =E_{Q^{*}}\left(\mathrm{e}^{\alpha C_{T \wedge \tau}(B, \alpha)} \mid \mathcal{G}_{t}\right) \geqslant \mathrm{e}^{\alpha E_{Q^{*}}\left(C_{T \wedge \tau} \mid \mathcal{G}_{t}\right)},
\end{aligned}
$$

其中不等式来自于 Jensen 不等式, 且 $X_{t, T \wedge \tau}\left(\vartheta^{*}\right) \geqslant 0$, 从而, 有

$$
C_{t}(B, \alpha) \geqslant E_{Q^{*}}\left(C_{T \wedge \tau}(B, \alpha) \mid \mathcal{G}_{t}\right) .
$$

由此可见可违约期权的指数效用无差异价值过程 $C(B, \alpha)$ 在最小熵鞅测度下为 $Q^{*}$ 上鞅, 由上鞅的 Doob-Meyer 分解

$$
C(B, \alpha)=C_{0}(B, \alpha)+M^{B}(\alpha)-A^{B}(\alpha)
$$


其中 $M^{B}(\alpha)$ 为 $\left(\mathbb{G}, Q^{*}\right)$ 鞅, $A^{B}(\alpha)$ 为适应可料增过程, 此处的上标 $B$ 表示与可违约期权 $B$ 有关. 由 于 $M^{B}(\alpha)$ 为 $\left(\mathbb{G}, Q^{*}\right)$ 鞅, $M^{B}(\alpha)$ 有 GKW (Galtchouk-Kunita-Watanabe) 分解, 存在有界 $\mathbb{R}^{d}$ 值 $\mathbb{G}$ 可 料过程 $\theta_{1}$ 使得

$$
M^{B}(\alpha)=\theta_{1} \cdot \widetilde{S}+q,
$$

其中 $q$ 为 $Q^{*}$ 局部鞅, 且与 $M(\alpha)$ 强正交, 又由于 $\widetilde{L}$ 为纯断鞅, 与连续鞅 $\widetilde{S}$ 强正交, 从而, $q$ 也有 $\mathrm{GKW}$ 分解, 存在有界 $\mathbb{R}^{d}$ 值 $\mathbb{G}$ 可料过程 $\theta_{2}$, 使得

$$
q=\theta_{2} \cdot \widetilde{L}+\widetilde{N}^{\perp},
$$

其中 $\widetilde{N}^{\perp}$ 与 $\widetilde{L}$ 强正交, 因此, $C(B, \alpha)$ 可以表示成

$$
C_{t}(B, \alpha)=C_{0}(B, \alpha)+\theta_{1} \cdot \widetilde{S}_{t}+\theta_{2} \cdot \widetilde{L}_{t}+\widetilde{N}_{t}^{\perp}-A_{t}^{B}(\alpha) .
$$

现在来考虑生成元 $f\left(t, \theta_{1}, \theta_{2}, \widetilde{N}_{t}^{\perp}\right)$ 的具体结构. 我们令

$$
\begin{aligned}
R_{t}^{\vartheta} & :=\exp \left\{\alpha\left(C_{t}(B, \alpha)-X_{t}(\vartheta)\right)\right\} \\
& =\exp \left\{\alpha C_{0}(B, \alpha)+\alpha\left(\theta_{1}-\vartheta^{\mathrm{T}} \varsigma\right) \cdot \widetilde{S}_{t}+\alpha\left(\theta_{2}-\vartheta^{\mathrm{T}} \zeta\right) \cdot \widetilde{L}_{t}+\alpha\left(\vartheta \cdot \widetilde{N}_{t}-\widetilde{N}_{t}^{\perp}\right)-\alpha A_{t}^{B}(\alpha)\right\} .
\end{aligned}
$$

对 $R_{t}^{\vartheta}$ 用 Itô 公式, 有

$$
\begin{aligned}
d R_{t}^{\vartheta}= & \alpha R_{t}^{\vartheta}\left\{\left(\theta_{1, t}-\vartheta_{t}^{\mathrm{T}} \varsigma_{t}\right) d \widetilde{S}_{t}+\left(\theta_{2, t}-\vartheta_{t}^{\mathrm{T}} \zeta_{t}\right) d \widetilde{L}_{t}+\left(\vartheta_{t} d \widetilde{N}_{t}-d \widetilde{N}_{t}^{\perp}\right)-d A_{t}^{B}(\alpha)\right\} \\
& +\frac{1}{2} \alpha^{2} R_{t}^{\vartheta}\left(\theta_{1, t}-\vartheta_{t}^{\mathrm{T}} \varsigma_{t}\right)^{\mathrm{T}} d\langle\widetilde{S}\rangle_{t}\left(\theta_{1, t}-\vartheta_{t}^{\mathrm{T}} \varsigma_{t}\right)+\frac{1}{2} \alpha^{2} R_{t}^{\vartheta} d\left\langle\left(\vartheta \cdot \widetilde{N}-\widetilde{N}^{\perp}\right)\right\rangle_{t} \\
& +R_{t}^{\vartheta}\left(\mathrm{e}^{\alpha\left(\theta_{2, t}-\vartheta_{t}^{\mathrm{T}} \zeta_{t}\right)}-1-\alpha\left(\theta_{2, t}-\vartheta_{t}^{\mathrm{T}} \zeta_{t}\right)\right)^{\mathrm{T}} \lambda_{t} 1_{t \leqslant \tau} d t \\
= & \alpha R_{t}^{\vartheta}\left\{\left(\theta_{1, t}-\vartheta_{t}^{\mathrm{T}} \varsigma_{t}\right) d \widetilde{S}_{t}+\left(\theta_{2, t}-\vartheta_{t}^{\mathrm{T}} \zeta_{t}\right) d \widetilde{L}_{t}+\left(\vartheta_{t} d \widetilde{N}_{t}-d \widetilde{N}_{t}^{\perp}\right)\right\} \\
& +\frac{1}{2} \alpha^{2} R_{t}^{\vartheta}\left(\theta_{1, t}-\vartheta_{t}^{\mathrm{T}} \varsigma_{t}\right)^{\mathrm{T}} d\langle\widetilde{S}\rangle_{t}\left(\theta_{1, t}-\vartheta_{t}^{\mathrm{T}} \varsigma_{t}\right)+\frac{1}{2} \alpha^{2} R_{t}^{\vartheta} d\left\langle\left(\vartheta \cdot \widetilde{N}-\widetilde{N}^{\perp}\right)\right\rangle_{t}-d A_{t}^{B}(\alpha) \\
& +R_{t}^{\vartheta}\left(\mathrm{e}^{\alpha\left(\theta_{2, t}-\vartheta_{t}^{\mathrm{T}} \zeta_{t}\right)}-1-\alpha\left(\theta_{2, t}-\vartheta_{t}^{\mathrm{T}} \zeta_{t}\right)\right)^{\mathrm{T}} \lambda_{t} 1_{t \leqslant \tau} d t .
\end{aligned}
$$

由引理 3.5 可知, $R_{t}^{\vartheta}$ 对于 $\vartheta \in \Theta$ 是 $Q^{*}$ 下鞅, 对于最优投资策略 $\vartheta^{*}$ 则为 $Q^{*}$ 鞅. 从而可知,

$$
\begin{gathered}
\frac{1}{2} \alpha^{2} R_{t}^{\vartheta}\left(\theta_{1, t}-\vartheta_{t}^{\mathrm{T}} \varsigma_{t}\right)^{\mathrm{T}} d\langle\widetilde{S}\rangle_{t}\left(\theta_{1, t}-\vartheta_{t}^{\mathrm{T}} \varsigma_{t}\right)+\frac{1}{2} \alpha^{2} R_{t}^{\vartheta} d\left\langle\left(\vartheta \cdot \widetilde{N}-\widetilde{N}^{\perp}\right)\right\rangle_{t}-R_{t}^{\vartheta} d A_{t}^{B}(\alpha) \\
+R_{t}^{\vartheta}\left(\mathrm{e}^{\alpha\left(\theta_{2, t}-\vartheta_{t}^{\mathrm{T}} \zeta_{t}\right)}-1-\alpha\left(\theta_{2, t}-\vartheta_{t}^{\mathrm{T}} \zeta_{t}\right)\right)^{\mathrm{T}} \lambda_{t} 1_{t \leqslant \tau} d t
\end{gathered}
$$

对 $\vartheta$ 为增过程, 对最优投资策略 $\vartheta^{*}$ 上式应该为零, 从而确保 $R_{t}^{\vartheta^{*}}$ 为鞅. 因此, 有

$$
\begin{aligned}
d A_{t}^{B}(\alpha)= & \underset{\vartheta \in \Theta}{\operatorname{ess} \inf }\left\{\frac{\alpha}{2}\left(\theta_{1, t}-\vartheta_{t}^{\mathrm{T}} \varsigma_{t}\right)^{\mathrm{T}} d\langle\widetilde{S}\rangle_{t}\left(\theta_{1, t}-\vartheta_{t}^{\mathrm{T}} \varsigma_{t}\right)+\frac{\alpha}{2} d\left\langle\left(\vartheta \cdot \widetilde{N}-\widetilde{N}^{\perp}\right)\right\rangle_{t}\right. \\
& \left.+\left(\frac{\mathrm{e}^{\alpha\left(\theta_{2, t}-\vartheta_{t}^{\mathrm{T}} \zeta_{t}\right)}-1-\alpha\left(\theta_{2, t}-\vartheta_{t}^{\mathrm{T}} \zeta_{t}\right)}{\alpha}\right)^{\mathrm{T}} \lambda_{t} 1_{t \leqslant \tau} d t\right\} .
\end{aligned}
$$

由于 $d \widetilde{S}_{t} \ll d t$, 从而意味着 $d \widetilde{N}, d \widetilde{N}^{\perp} \ll d t$, 令 $f\left(t, \theta_{1}, \theta_{2}, \widetilde{N}^{\perp}\right) d t:=d A_{t}^{B}(\alpha)$, 有

$$
\begin{aligned}
f\left(t, \theta_{1}, \theta_{2}, \widetilde{N}^{\perp}\right) d t= & \operatorname{essinf}_{\vartheta \in \Theta}\left\{\frac{\alpha}{2}\left(\theta_{1, t}-\vartheta_{t}^{\mathrm{T}} \varsigma_{t}\right)^{\mathrm{T}} d\langle\widetilde{S}\rangle_{t}\left(\theta_{1, t}-\vartheta_{t}^{\mathrm{T}} \varsigma_{t}\right)+\frac{\alpha}{2} d\left\langle\left(\vartheta \cdot \widetilde{N}-\widetilde{N}^{\perp}\right)\right\rangle_{t}\right. \\
& \left.+\left(\frac{\mathrm{e}^{\alpha\left(\theta_{2, t}-\vartheta_{t}^{\mathrm{T}} \zeta_{t}\right)}-1-\alpha\left(\theta_{2, t}-\vartheta_{t}^{\mathrm{T}} \zeta_{t}\right)}{\alpha}\right)^{\mathrm{T}} \lambda_{t} 1_{t \leqslant \tau} d t\right\} .
\end{aligned}
$$

由引理 3.6 可知, 上式的解存在. 从而可知, $C(B, \alpha)$ 是方程的解. 解的唯一性由命题 3.3 可得. 


\section{4 结论}

本文首先考虑一个信用敏感的资产, 通过流的扩张和文献 [27, 引理 2.4 和定理 3.1$]$, 使得该风险资 产成为一个 $\mathbb{G}$ 局部鞅, 避免了需要作出通常可违约未定权益定价文献中的 $\mathbb{F}$ 鞅 (局部鞅) 为 $\mathbb{G}$ 鞅 (局 部鞅) 的假设. 然后利用指数效用最大化的方法给出可违约期权的指数效用无差异价值过程的导向随 机微分方程, 指出了该方程的生成元的具体结构, 并证明了方程的解的存在性和唯一性, 从而解决了 不完备市场条件下的可违约期权的定价问题.

\section{参考文献}

1 Schweizer M. On the minimal martingale measure and the Follmer-Schweizer decomposition. Stoch Anal Appl, 1995, 13: $573-599$

2 Schweizer M. Approximation pricing and the variance-optimal martingale measure. Ann Probab, 1996, 24: 206-236

3 Delbaen F, Schachermayer W. The variance-optimal martingale measure for continuous processes. Bernoulli, 1996, 2: $81-105$

4 Arai T. An extension of mean-variance hedging to the discontinuous case. Finance Stoch, 2005, 9: 129-139

5 Cerny A, Kallsen J. On the structure of general mean-variance hedging strategies. Ann Probab, 2007, 35: 1479-1531

6 Zhou X, Li D. Continuous-time mean-variance portfolio selection: A stochastic LQ framework. Appl Math Optim, 2000, 42: 19-33

7 Lim A E B, Zhou X Y. Mean-variance portfolio selection with random parameters. Math Oper Res, 2002, 27: 101-120

8 Kohlmann M, Tang S J. Multidimensional backward stochastic Riccati equations and applications. SIAM J Control Optim, 2003, 41: 1696-1721

9 Bobrovnytska O, Schweizer M. Mean-variance headging and stochatic control: Beyond the Brownian setting. IEEE Trans Automat Control, 2004, 49: 396-408

10 Kohlmann M, Xiong D W, Ye Z X. Mean variance hedging in a general jump model. Appl Math Finance, 2010, 17: $29-57$

11 Jeanblanc M, Mania M, Santacorce M, et al. Mean-variance hedging via stochastic control and BSDEs for general semimartingales. Ann Appl Probab, 2012, 22: 2388-2428

12 Xiong D W, Kohlmann M. Optimal exponential utility in a jump bond market. Stoch Anal Appl, 2011, 29: 78-105

13 Hodges S, Neuberger A. Optimal replication of contingent claim under transaction costs. Rev Futures Markets, 1989, 8: $222-239$

14 Rouge R, El Karoui N. Pricing via utility maximization and entropy. Math Finance, 2000, 10: 259-276

15 Musiela M, Zariphopoulou T. An example of Indifference prices under exponential preferences. Finance Stoch, 2004, 8: $229-239$

16 Mania M, Santacroce M, Tevzadze R. A semimartingale BSDE related to the minimal entropy martingale measure. Finance Stoch, 2003, 7: 384-402

17 Mania M, Schweizer M. Dynamic exponential utility indifference valuation. Ann Appl Probab, 2005, 15: 2113-2143

$18 \mathrm{Hu}$ Y, Imkeller P, Muller M. Utility maximization in incomplete markets. Ann Appl Probab, 2005, 15: 1691-1712

19 Morlais M. Utility maximization in a jump market model. Stochastics, 2009, 81: 1-27

20 Xiong D, Kohlmann M. Opimal exponential utility in a jump bond market. Stoch Anal Appl, 2011, 29: 78-105

21 Bielecki T R, Björk T, Jeanblanc M, et al. Hedging of defaultable claims. In: Paris-Princeton Lecture on Mathematical Finance 2003. Lecture Notes in Mathematics. New York: Springer, 2004, 1-132

22 Biagini F, Cretarola A. Quadratic hedging methods for defaultable claims. Appl Math Optim, 2007, 56: 425-443

23 Kohlmann M, Xiong D. The mean-variance hedging of a defaultable option with partial information. Stoch Anal Appl, 2007, 25: 869-893

24 Kharroubi I, Lim T, Ngoupeyou A. Mean-variance hedging on uncertain time horizon in a market with a jump. ArXiv:1206.3693, 2013

25 Jeanblanc M, Le Cam Y. Immersion property and credit risk modelling. In: Optimality and Risk-Modern Trends in Mathematical Finance. New York: Springer, 2010, 99-132

26 Delia C, Jeanblanc M, Nikeghbali A. Default times, no-arbitrage conditions and change of probability measures. Finance Stoch, 2012, 16: 513-535 
27 Jeanblanc M, Le Cam Y. Progressive enlargement of filtrations with initial times. Stochastic Process Appl, 2009, 119: $2523-2543$

28 何声武, 汪嘉岗, 严加安. 半鞅与随机分析. 北京: 科学出版社, 1995

29 Aumann R. Measurable utility and the measurable choice theorem. In: Guilbaud G T, ed. La Decision. Paris: Editions du Centre National de la Recherche Scientifique, 1969, 15-26

\title{
A pricing defaultable claim model based on exponential utility indifference value process in an incomplete market
}

WANG KaiMing, ZHANG HuiYan \& YAO Jin

\begin{abstract}
We study the dynamics of exponential utility indifference value process $C_{t}(B, \alpha)$ for a defaultable claim in an incomplete market. Other than most of papers dealing with defautable claim, we do not assume the hypothesis of invariance of martingale that is $\mathcal{H}$ hypothesis. We firstly transform the default sensitive assets $S$ to be a local martingale through progressively enlargement of filtration and changes of measure. Then, we introduce a specific backward stochastic differential equation (BSDE) and prove the existence and uniqueness of the solution. Finally, we prove the exponential utility indifference value process $C_{t}(B, \alpha)$ is the solution to the BSDE based on the fact that $C_{t}(B, \alpha)$ is a supermartingale for investment strategies and a martingale for optimal strategy under the minimal entropy martingale measure.
\end{abstract}

Keywords defaultable claim, exponential utility indifference value process, default sensitive assets, backward stochastic differential equation

$\operatorname{MSC}(2010) \quad 60 \mathrm{H} 15,91 \mathrm{~B} 25,91 \mathrm{G} 40$

doi: $10.1360 / 012012-547$ 